\title{
Key findings of the US Cystic Fibrosis Foundation's clinical practice benchmarking project
}

\author{
Michael P Boyle, ${ }^{1}$ Kathryn A Sabadosa, ${ }^{2}$ Hebe B Quinton, ${ }^{2}$ \\ Bruce C Marshall, ${ }^{3}$ Michael S Schechter ${ }^{4}$
}

- Additional material is published online only. To view please visit the journal online (http://dx.doi.org/10.1136/bmjas2013-002369)

${ }^{1}$ Department of Medicine, Johns Hopkins University School of Medicine, Baltimore,

Maryland, USA

${ }^{2}$ The Dartmouth Institute for Health Policy and Clinical

Practice, Lebanon, New Hampshire, USA

${ }^{3}$ Cystic Fibrosis Foundation, Bethesda, Maryland, USA

${ }^{4}$ Department of Pediatrics, Virginia Commonwealth University, Richmond, Virginia, USA

\section{Correspondence to} Dr Michael P Boyle, Division of Pulmonary and Critical Care Medicine, Johns Hopkins, 1830 E Monument Street, 5th floor Baltimore, MD 21205 , USA; mboyle@jhmi.edu

Received 27 July 2013 Revised 6 December 2013 Accepted 13 December 2013

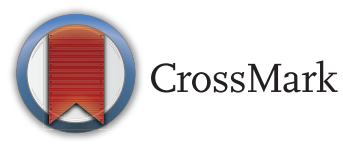

To cite: Boyle MP, Sabadosa KA, Quinton HB, et al. BMJ Qual Saf 2014;23: i15-i22.

\begin{abstract}
Benchmarking is the process of using outcome data to identify high-performing centres and determine practices associated with their outstanding performance. The US Cystic Fibrosis Foundation (CFF) Patient Registry contains centrespecific outcomes data for all CFF-certified paediatric and adult cystic fibrosis (CF) care programmes in the USA. The CFF benchmarking project analysed these registry data, adjusting for differences in patient case mix known to influence outcomes, and identified the topperforming US paediatric and adult CF care programmes for pulmonary and nutritional outcomes. Separate multidisciplinary paediatric and adult benchmarking teams each visited 10 CF care programmes, five in the top quintile for pulmonary outcomes and five in the top quintile for nutritional outcomes. Key practice patterns and approaches present in both paediatric and adult programmes with outstanding clinical outcomes were identified and could be summarised as systems, attitudes, practices, patient/family empowerment and projects. These included: (1) the presence of strong leadership and a well-functioning care team working with a systematic approach to providing consistent care; (2) high expectations for outcomes among providers and families; (3) early and aggressive management of clinical declines, avoiding reliance on 'rescues'; and (4) patients/families that were engaged, empowered and well informed on disease management and its rationale. In summary, assessment of practice patterns at CF care centres with top-quintile pulmonary and nutritional outcomes provides insight into characteristic practices that may aid in optimising patient outcomes.
\end{abstract}

\section{BACKGROUND}

In an effort to optimise care for individuals with cystic fibrosis (CF), the US CF Foundation (CFF) has established a national network of over 200 care centres that all contribute data to a national $\mathrm{CFF}$ patient registry (CFFPR). This registry contains detailed information on each individual CF centre's treatment practices, patient demographics, and pulmonary and nutritional outcomes dating back to the mid 1980s. ${ }^{1}{ }^{2}$ While the initial intent of the CFFPR was to obtain descriptive information on the population of individuals with $\mathrm{CF}$, it quickly became evident that much could also be learned about CF care centres themselves. There was significant variability in routine clinical practices among care centres, including those for which standardised treatment practice guidelines had been established. ${ }^{1}$ In addition, even after adjustment for differences in patient demographics, socioeconomic status and other uncontrollable variations in case mix, ${ }^{3}$ there were significant disparities between centres in key patient outcomes such as lung function and nutritional status. ${ }^{3}$ Previous analysis using the CFFPR had also noted that being cared for at a 'top 10' CF centre resulted in an $\sim 7$ year longer survival compared with receiving care at all other CF care centres in the network.

While initially disconcerting, this significant variability in practice patterns and outcomes between CF centres offered a great opportunity to identify clinical practices associated with best CF outcomes. One method for potentially identifying these key clinical practices was 'benchmarking' - that is, utilising outcome data to identify top-performing centres, and then comparing practice patterns at those centres with all other centres. This strategy has been used for decades in business administration to identify best practices and encourage spread of those practices throughout an organisation. ${ }^{5}$ Systematic 
use of benchmarking strategy to identify best practices in clinical healthcare has been a more recent development however. ${ }^{6}$

A benchmarking analysis has transformed CF care once in the past. In a 1988 publication, Corey et $\mathrm{al}^{7}$ contrasted nutritional indices and survival in Boston versus Toronto between 1972 and 1981 and showed superior height, weight and survival in Toronto, where the traditional restriction of dietary fat had been replaced by an alternative approach encouraging high-fat and high-calorie feeding. This classic paper was instrumental in changing nutritional practice throughout the CF community by drawing attention to the advantage of the Toronto approach.

More than a decade later, two analyses of data from the Epidemiologic Study of CF (ESCF) identified care centres with lung function in the highest quartile and compared reported care practices at those centres with those in the lowest quartile. Johnson et al ${ }^{8}$ reported that high-quartile sites performed more clinic visits, spirometry and airway cultures across all age groups, as well as more and longer courses of intravenous antibiotics. A separate study that focused on the care of young children found that centres in the highest quartile for lung function in 6-8-year-olds performed more airway cultures and prescribed more oral corticosteroids. ${ }^{9}$

While registries such as the CFFPR and ESCF can be very effective for identifying which centres have outstanding outcomes, they are limited in their ability to allow identification of practice patterns associated with best outcomes. Only variables available in the database can be studied, and the majority of daily practices and attitudes that characterise a successful care team are not captured. Deeper insights are potentially available if investigators make more extensive explorations of processes by physically visiting highachieving centres. ${ }^{10}$

In an effort to systematically document clinical practices resulting in the best outcomes for individuals with $\mathrm{CF}$, the CFF organised investigative 'benchmarking teams' made up of representatives from different disciplines and different CF care centres to visit topperforming centres. Separate adult and paediatric benchmarking teams were created, as most CF centres have separate multidisciplinary paediatric and adult care clinical teams ('programmes') with expertise and unique practice patterns suited for their patient group. After assessing outcome data from the CFFPR to identify appropriate sites, these benchmarking teams visited the top-performing paediatric and adult care programmes and used a combination of questionnaires, interviews and observation to thoroughly explore clinical care processes at benchmark programmes.

The purpose of this article is to (1) describe the methods used to determine top-performing programmes, conduct benchmarking visits, and assess practice patterns and (2) identify and discuss the key practices and treatment approaches shared by $\mathrm{CF}$ programmes with exceptionally good clinical outcomes.

\section{METHODS FOR SELECTION OF TOP-PERFORMING PROGRAMMES FOR BENCHMARKING VISITS}

We used CFFPR data to separately identify the paediatric and adult CF programmes caring for 40 or more patients with consistently outstanding pulmonary and nutrition outcomes over the 5-year period from 2001 to 2005. Pulmonary and nutritional outcomes were selected, as they are key parameters in CF care because of their association with survival and quality of life. ${ }^{11}{ }^{12}$ Corrected median forced expiratory volume in $1 \mathrm{~s}$ percent predicted $\left(\mathrm{FEV}_{1} \%\right)$ was used as the primary pulmonary outcome measure for both paediatric and adult $\mathrm{CF}$ programmes, and body mass index percentile (BMI\%) and body mass index (BMI) were used as primary nutritional outcomes for paediatric and adult care programmes, respectively. ${ }^{13}$ To control for differences between programmes in patient demographics that are known to affect outcomes, programme measures were case-mix-adjusted for age, gender, use of pancreatic enzymes, median family income and race/ethnicity. ${ }^{3}$ Paediatric analysis included examination of outcomes by separate age strata: ages 2-11, 6-12 and 13-17. Corrections were also made for the adult programme analysis to adjust for the effect of paediatric care in years before transition. Further details on methods used to assess and select top-performing programmes are available in the online supplementary appendix.

The final product for both paediatric and adult analyses was an ordered ranking of CF programmes by corrected outcome for each of the previous 5 years (figures 1-3). We then selected paediatric and adult programmes that were consistently in the top quintile for either pulmonary or nutritional outcomes over the entire 5 year period. From this list, we chose programmes of different sizes and geographic location, to ensure a representative sampling, and programmes with stable staffing over the previous 5 years, to ensure that current practices reflected those during the assessed time period. A total of 20 care programmes were selected for benchmarking visits: 10 paediatric and 10 adult, with five in each age group in the top quintile for pulmonary outcomes and five in the top quintile for nutritional outcomes (figures 1-3).

\section{METHODS FOR ASSESSING PRACTICE PATTERNS AT BENCHMARKING VISITS}

Separate paediatric and adult teams of CF experts with training in systems-oriented approaches to care were formed. Each of these benchmarking teams consisted of six members from different CF care programmes, including a physician, nurse, dietician, respiratory therapist, social worker and CF parent. 
Median FEV1 Percent Predicted for Patients 6 to 12

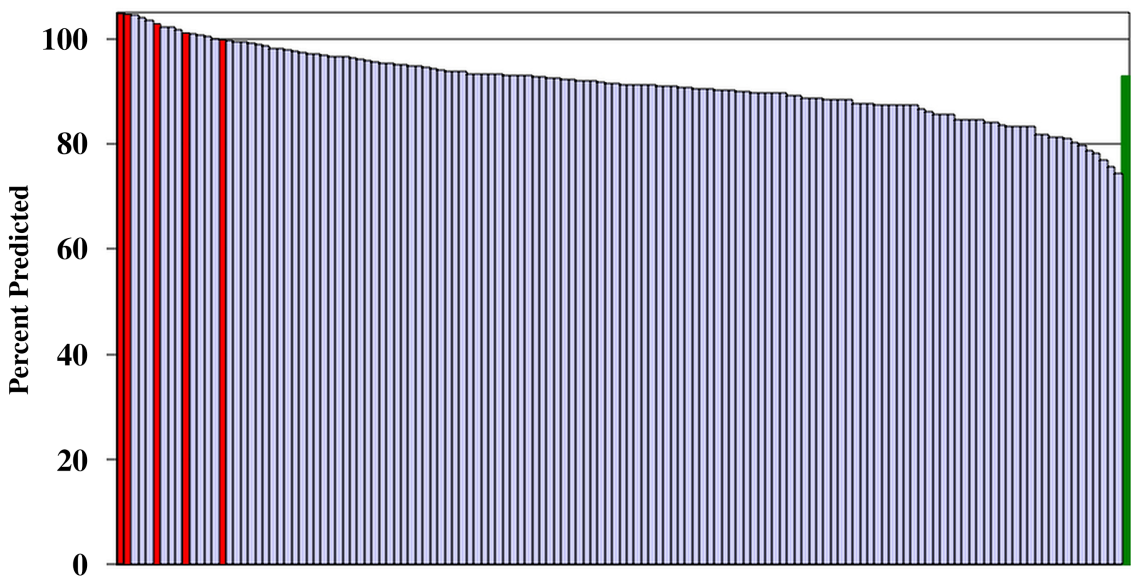

Figure 1 Median corrected forced expiratory volume in $1 \mathrm{~s}$ percent predicted ( $\mathrm{FEV}_{1} \%$ ) of children age 6-12 at US Paediatric Care Programs, 2004. Each bar represents a separate paediatric care programme caring for more than 50 patients. Best quarterly FEV 1 for each patient used, with $\mathrm{FEV}_{1}$ adjusted for differences among programmes in patient age, gender, use of pancreatic enzymes, race/ethnicity, and income from zip code. Those programmes highlighted in red represent sites where paediatric respiratory benchmarking visits occurred. Multiple age strata and results over 5 years were used in selecting sites.

From 2006 to 2007, the paediatric benchmarking team visited the 10 selected paediatric programmes, and the adult benchmarking team visited the 10 adult programmes. At each site visit, the benchmarking team was accompanied by a volunteer multidisciplinary team from a CF care programme that expressed an interest in visiting a high-performing programme (called the 'visiting team'). To aid in identification of key treatment practices, a comprehensive systems-oriented questionnaire evaluating details of clinical practice was completed by the host programme.

Programme visits generally lasted $24 \mathrm{~h}$, and began with a preclinic dinner during which the host team would be asked standard questions by the participants to aid in identification of key treatment practices.

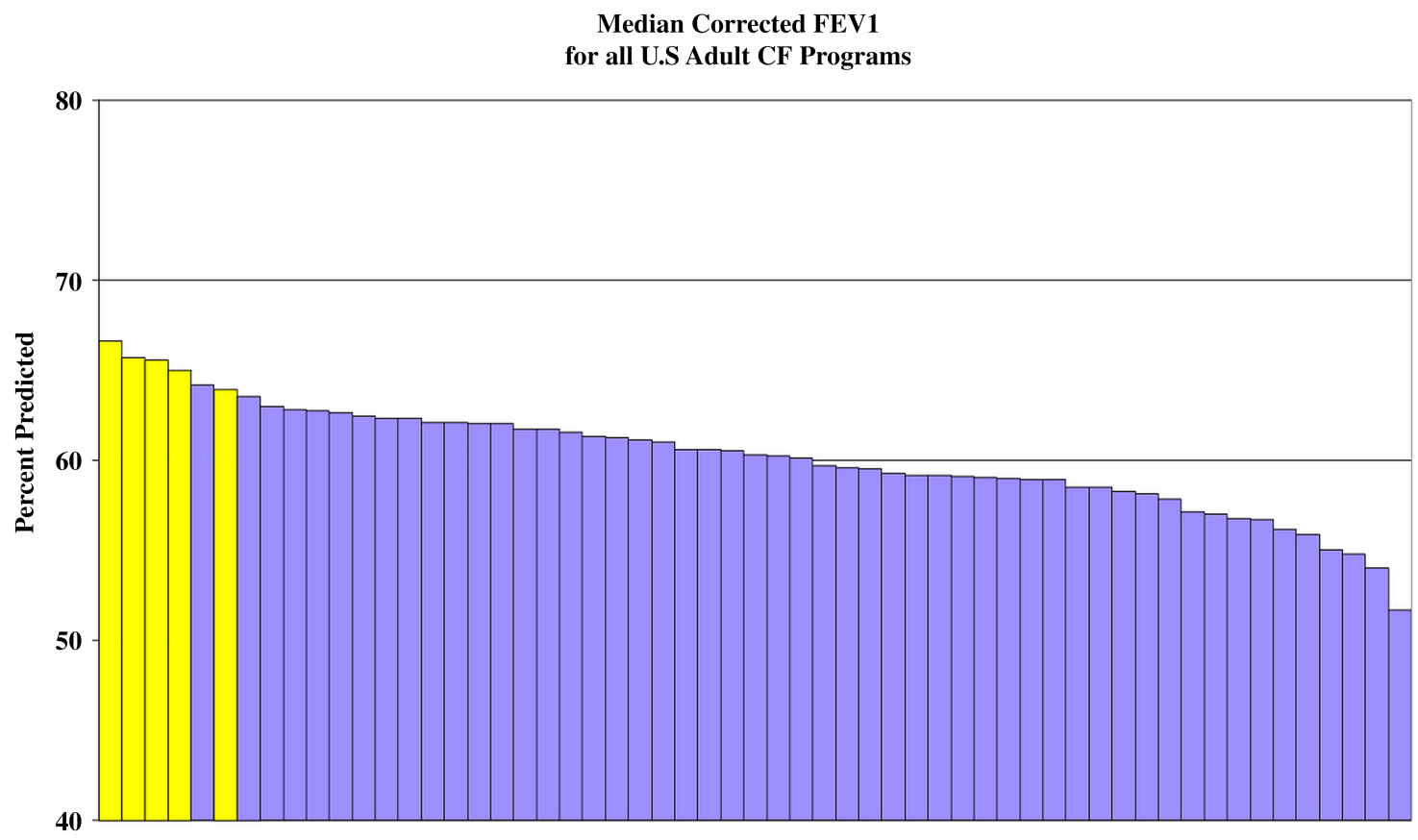

Figure 2 Median corrected forced expiratory volume in $1 \mathrm{~s}$ percent predicted (FEV1\%) of adults $>24$ years of age at US Adult Care Programs, 2005. Each bar represents a separate adult care programme caring for more than 40 patients. Lung transplant patients censored. Best annual FEV 1 for each patient used, with $\mathrm{FEV}_{1}$ adjusted for differences among programmes in patient age, gender, use of pancreatic enzymes, race/ethnicity, income from zip code, and FEV 7 years ago. Those programmes highlighted in yellow represent sites where adult respiratory benchmarking visits occurred. 


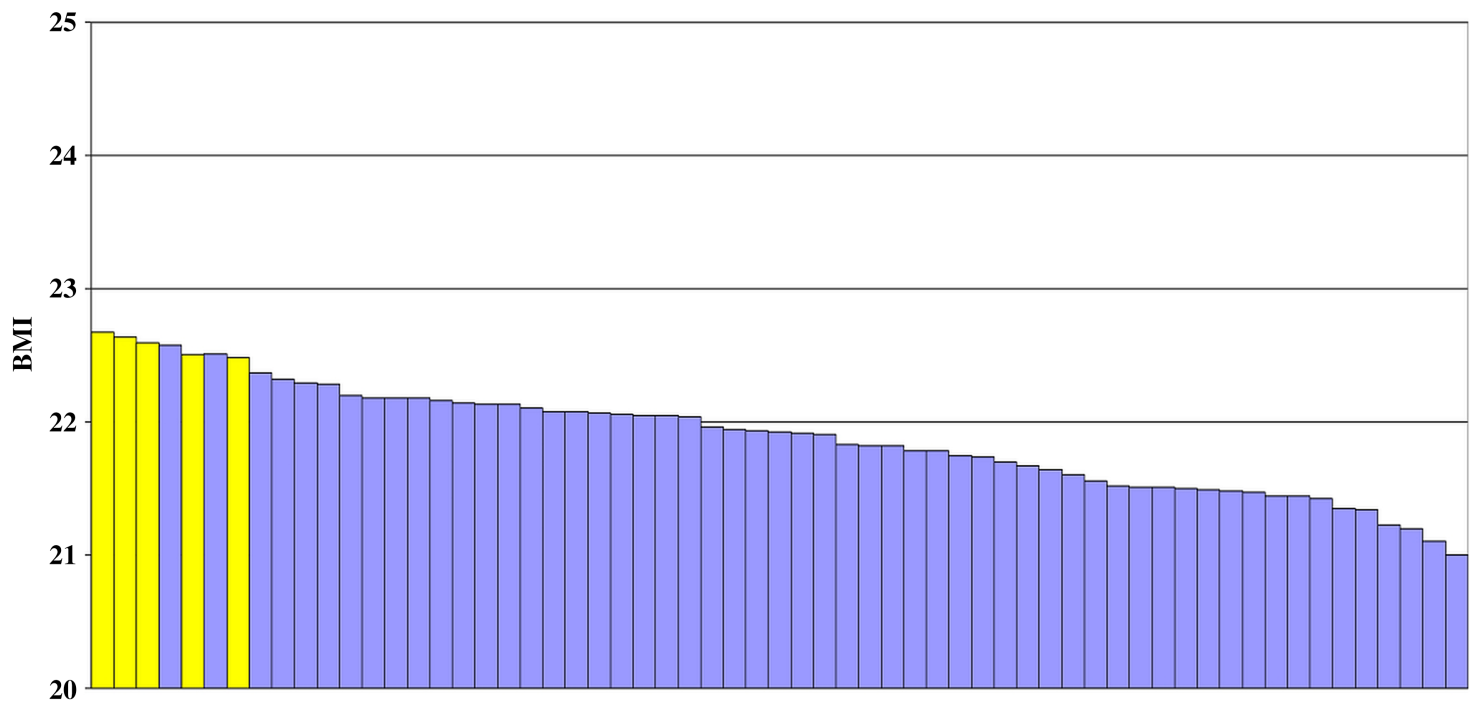

Figure 3 Median corrected body mass index (BMI) of adults $>24$ years of age at US Adult Care Programs, 2005. Each bar represents a separate adult care programme caring for more than 40 patients. Lung transplant patients censored. BMI adjusted for differences among programmes in patient age, gender, use of pancreatic enzymes, race/ethnicity, income from zip code, and BMI 7 years ago. Those programmes highlighted in yellow represent sites where adult nutritional benchmarking visits occurred.

Teams would compare programme-specific registry reports of clinical practices and focus on differences in their approaches. This discussion would lead to the development of hypotheses regarding practice differences and set a framework for observations in clinic. During clinic, team members were paired by discipline to observe and discuss care strategies. The parent representative interviewed patients and families. A hospital visit was made which focused on the processes of inpatient care. When possible, the benchmarking and visiting teams sat in on host team meetings to observe interactions among team members. At the conclusion of the programme visit, benchmarking and visiting team members met and first individually summarised their conclusions about unique practice patterns. The benchmarking team then developed a consensus on the key practice patterns and approaches from the programme and listed them in a summary of the visit.

As a final tool to aid in identifying key CF clinical practices associated with outstanding outcomes, host care programmes subsequently completed a 65 question survey based on initial observations of practice patterns from programme visits and their responses were compared with those from low-performing care programmes across the USA.

\section{FINDINGS FROM BENCHMARKING VISITS ON KEY PRACTICE PATTERNS OF HIGH-PERFORMING PROGRAMMES}

There were five key practice patterns and approaches identified in both paediatric and adult programmes with outstanding clinical outcomes. These can be summarised as systems, attitudes, practices, patient/ family empowerment and projects (SAPPP) (box 1).

\section{Systems}

Systems at high-performing programmes were usually characterised by the presence of a programme director and clinic coordinator with strong leadership skills and a dedicated and experienced multidisciplinary care team. At preclinic meetings and during clinic, these teams demonstrated positive team dynamics, including good communication and cooperation among team members, and usually had adequate team personnel levels compared with national averages. Visiting teams noted that delivery of treatment was streamlined and efficient: responsibilities were clear, with little 'falling through the cracks'; team members could anticipate and support what recommendations would be made; decisions were likely to be made and communicated without delay; and patients and families could be effectively and consistently educated by team members about the rationale for treatments. The adult benchmarking team noted a history of close ties between paediatric and adult caregivers. Another aspect of systems at these high-performing programmes was a clinical organisation that permitted close tracking of patient clinical details and outcomes. Finally, top-performing programmes used telephone contact as a key part of their management strategy: staying in close contact with patients and families, advancing care without always requiring a clinic visit, and being readily accessible by phone to allow rapid engagement with the care team at times of health concerns. 
Box 1 Key characteristics of programmes with topquintile cystic fibrosis clinical outcomes

Systems

1. Strong leadership

2. Dedicated multidisciplinary team

3. Easy accessibility by patients and families

4. Close tracking of clinical details and outcomes

Attitudes

1. High expectations for pulmonary and nutritional status

2. Low threshold for vigorous treatment of health declines

3. Aggressive use of antibiotics for pulmonary symptoms

4. Team consensus on standard approach to care

Practices

1. Preclinic review of patients and treatment planning

2. More frequent clinic visits for identified health concerns

3. Regular patient visits with full multidisciplinary team

Patient/family engagement and empowerment

1. Patients provided with data on their clinical outcomes

2. Patients/families educated on high outcome expectations and need for early, aggressive intervention for declines

3. Patients/families encouraged to provide feedback on their clinical care experiences and concerns

Projects

1. Self-assess programme outcomes and practice patterns

2. Develop projects to improve programme performance of key clinical practices

\section{Attitudes}

Attitudes were characterised by high expectations for what was achievable and acceptable pulmonary or nutritional status. Top-performing programmes almost uniformly described having a low threshold for treating any decline from baseline. This attitude was reflected both in the clinical team approach and in the attitudes of patients and families. Programmes benchmarked for outstanding BMI endorsed the attitude that nutritional status was just as important an outcome measure to follow as $\mathrm{FEV}_{1}$, and consistently discussed strategies for gaining weight at clinic visits with all patients who were below the target BMI identified by CFF consensus guidelines. ${ }^{14}$ Programmes benchmarked for $\mathrm{FEV}_{1}$ articulated a willingness to treat with antibiotics after only a few days of persistent symptoms and downplayed concerns about development of antimicrobial resistance. The paediatric benchmarking team frequently found an attitude best described as 'intentional consensus'-value placed on trying to develop a standard approach to care that would be used by all providers at the clinic. This standard approach was usually arrived at through formal meetings designed to develop consensus.

\section{Practices}

Practices that were common among programmes with outstanding outcomes included having a preclinic meeting or assessment of patients. Teams noted that reviewing clinical details and formulating treatment plans before clinic prevented missing important follow-up issues during the rush of clinic. Another frequently used strategy in high-performing programmes was involving patients in their care by regularly giving them feedback on their outcomes. One common way of doing this was by sharing a graph (for instance, of $\mathrm{FEV}_{1}$ or BMI), growth chart or picture (such as chest CT). In addition, high-performing programmes endorsed more frequent follow-up visits as a particularly effective strategy to address specific areas of concern, encourage adherence, and provide accountability. Recognising the desirability of a prompt response to small changes in clinical status, these programmes were likely to have policies that facilitated seeing patients at short notice if needed. When patients were seen in clinic, all team members, especially dieticians and physical and respiratory therapists, were likely to see the patients-rather than just 'as needed'. This helped to reinforce the essential basics of CF care at each visit rather than only focusing on problem issues.

\section{Patient/family engagement and empowerment}

The paediatric team consistently found that patients and families at the benchmarked programmes were engaged and empowered and were seen as active participants on the care team. They tended to be well informed on disease management and its rationale and were taught to have high expectations. Specifically, they understood when it was appropriate to contact the CF programme, and furthermore they could anticipate what actions would be taken when they called or were seen in clinic. Their expectations provided additional assurance that they would receive an appropriate response in a timely manner. Parents of paediatric patients often participated in an advisory capacity at high-performing paediatric programmes or were engaged in development and conduct of family education and support groups.

High-performing adult care programmes also encouraged their patients to be active participants in their healthcare decisions and view themselves as part of their care team. Adult programmes were less likely to have adult patients involved in development and conduct of education and support groups, citing infection-control concerns as a limiting factor. Many of these programmes endorsed instead the practice of regularly engaging their patients outside of clinic through the use of listservs or simple emails. 


\section{Projects}

Projects to improve delivery of care were often found in the benchmarked adult programmes. These projects were usually a result of a culture of ongoing selfassessment that led to identification of programme shortcomings, accompanied by a determination to improve on these shortcomings. Many of these projects were informal, but others included careful analysis of outcomes and practice patterns to identify areas in need of improvement. While some of these projects focused on improving clinical outcomes such as nutritional status, others focused on optimising delivery of care (such as following treatment guidelines).

\section{BENCHMARKING FOLLOW-UP SURVEY}

Many of the conclusions about key attitudes and practices observed in benchmarked centres were confirmed in a post-benchmarking survey taken by a larger group of adult programmes identified to be in the top and bottom national quartiles for $\mathrm{FEV}_{1}$ and BMI. The responses provided further objective evidence that centres with best clinical outcomes are more likely to intervene early and aggressively for declines in health. Top-quartile programmes for $\mathrm{FEV}_{1}$ were statistically significantly more likely to start early antibiotics for persistent respiratory symptoms (3.7 vs 8.7 days), use antibiotics to treat 'common cold symptoms', and not be afraid that antibiotic use would 'lead to development of resistance'. A similar aggressive approach was noted in programmes with best nutrition outcomes: top-quartile programmes for BMI scored significantly higher for agreeing with the statement 'small declines in weight or BMI prompt immediate reaction in our program'.

\section{DISCUSSION}

One of the advantages of a well-constructed patient registry such as the CFFPR is the opportunity it offers to compare outcomes and practice patterns across care programmes. Significant variability between programmes suggests differences in the efficiency of delivery of care and offers the opportunity to gain knowledge of achievable outcomes and the care practices associated with best outcomes. Benchmarking has potential to aid in the transformation of care delivery by allowing caregivers to: learn new ways of working from those who have achieved excellence; share knowledge about overcoming common problems; set realistic targets for improvement; and develop a culture of willingness to learn from outside one's own programme. ${ }^{15}$

The findings of the CFF's paediatric and adult benchmarking teams on the practices, patterns and attitudes that characterise high-performing CF programmes were remarkably similar in that they both found several 'signature themes'. One theme was the presence of an efficiently functioning care team working with a well-thought-out systematic approach to providing consistent care. Some specific strategies supporting this theme included preclinic preparation to ensure ideal use of clinic time and avoid overlooking essential details of care, and extensive use of the telephone to make the team (a) capable of rapidly instituting care plans and (b) readily accessible to patients. Additional signature themes at benchmarked centres included high expectations for outcomes among providers and families and early and aggressive management for declines. This meant avoiding reliance on 'rescues' for treatment, but instead making early use of antibiotics or nutritional interventions upon any sign of decline. This approach was also characterised by increased frequency of clinic visits in patients with pulmonary or nutritional declines. Another signature theme was patients/families who were engaged, empowered and well informed on disease management and its rationale. Centres worked to engage patients and families as care partners by providing them with feedback tools such as graphs or images to track their own outcomes. The adult benchmarking team also noted that many high-performing programmes had a culture of ongoing self-assessment that led to identification of programme shortcomings and development of projects to improve performance.

Although not easily quantifiable, high-performance teams usually demonstrated a pride and enthusiasm for their work that was readily apparent to visiting teams. This would be consistent with previous observations by Huber and colleagues that a positive working environment and professional satisfaction were fundamental characteristics of high-performance healthcare teams. ${ }^{16}$ While fostering a strong team identity, high-performance programmes also encouraged development of individual expertise and professional success for each team member.

In addition to educating about characteristics of high-performance CF care teams, the CFF benchmarking project also imparted lessons for future healthcare performance assessments. One aspect of this project that worked particularly well and should be considered for future healthcare benchmarking projects was the use of a multidisciplinary team to carry out the benchmarking visits. Pairing of caregivers within specialties allowed focus on specific areas of unique practice and expertise. The presence of a multidisciplinary team also encouraged greater analysis of the positive intra-team dynamics that were usually so apparent at high-performing programmes.

One other strategy that worked well in this project but could be an even greater emphasis in future projects was the ongoing development of hypotheses about key practices associated with outstanding outcomes. By continually formulating a cumulative hypothesis of key practices, each successive benchmarking visit can offer an independent opportunity to test validity. 
One cautionary note that must be highlighted regarding benchmarking is the importance of developing a valid process to identify high-performance programmes. For $\mathrm{CF}$ this required using case-mix adjustment for differences in the distribution of demographic determinants of outcomes including age, ethnicity, pancreatic status and socioeconomic status. ${ }^{3}$ Along with case-mix issues, there is the challenge of agreeing on and determining the best measures of performance. ${ }^{4}$ Finally, comparisons of adult programmes must take into account the fact that they inherit different starting baselines of disease status from their feeder paediatric programme practices. This benchmarking project attempted to utilise the most rigorous methods available for identifying top-performing programmes and control for differences between programmes in patient case mix.

As rigorous as the initial statistical analysis was for identifying top-performing programmes, one thing that we would do differently in future benchmarking projects would be to use more rigorous methods of qualitative analysis to evaluate the benchmarking visits themselves. Conclusions from visits were drawn by first having each benchmarking team member individually make a list of observed practice patterns, and then utilising a team meeting to present findings and reach consensus on key practices. More rigorous qualitative research methodologies, including formal taped interviews and analysis of transcripts, would further strengthen conclusions for future projects.

The ultimate goal of healthcare benchmarking is assimilation of identified key practices throughout the healthcare community. This may be done individually by single programmes performing self-assessment for key practices and instituting change as needed, or through organised small-group, quality-improvement, learning collaboratives that provide support for programme self-assessment and continuous improvement. These strategies have led to measurable improvements in outcomes at numerous CF care programmes, and can be a model for the CF community as we seek to continue to improve clinical outcomes in upcoming years. ${ }^{17-21}$

One unique model of group improvement is the German CF Quality Assurance project, which has specifically aimed to develop clinical goals for its participating programmes based on benchmarks derived from registry data. ${ }^{22-25}$ Quality indicators were selected (airway cultures free of Pseudomonas aeruginosa, nutritional measures, lung function, and lack of serious complications). During two annual conferences, the highest-ranking programmes for these indices presented their treatment strategies, and the ensuing discussions led to the identification of clinical practices that programmes would aspire to adopt. ${ }^{23}$

Efforts to improve CF outcomes through the development of new therapeutics will likely always be a focus of the CF caregiver community. But the findings of this benchmarking project offer the CF community additional potential avenues for improving clinical outcomes by optimising delivery of currently available therapies by adapting key attitudes and practices. With the emerging development of CF registries allowing identification of programmes worldwide with outstanding outcomes, the door is just opening to the possibility of international comparisons and efforts for identifying and sharing effective approaches to CF care.

Acknowledgements Members of the paediatric benchmarking team: Stacy Bichl, APN-CPNP, Ann \& Robert H. Lurie Children's Hospital of Chicago, Chicago, IL, USA; Amanda Leonard, MPH, RD, CDE, Johns Hopkins University School of Medicine, Baltimore, MD, USA; John Nash, LMSW, Albany Medical Center, Albany, NY, USA; Kathleen Richards, RRT, RCP, Primary Children's Medical Center, Salt Lake City, UT, USA. Members of the adult benchmarking team: Julie Haas, RD, CD, Medical College of Wisconsin, Milwaukee, WI, USA; Karen Hollen, MSW, CSW, University of Kentucky, Lexington, KY, USA; Mary Lester, RRT, RCP, Medical University of South Carolina, Charleston, SC, USA; Christine Singh, MSN, CNP, Akron Children's Hospital, Akron, OH, USA.

Contributors MPB performed the study, collected and analysed data, and drafted and revised the manuscript. He is the guarantor. KAS and BCM helped design and perform the study and reviewed and approved the manuscript. HBQ helped with study design, performed the data analysis, and reviewed and approved the manuscript. MSS performed the study, collected and analysed data, and drafted and revised the manuscript.

Funding US Cystic Fibrosis Foundation (CFF BOYLE09QI0).

Competing interests None.

Provenance and peer review Not commissioned; externally peer reviewed.

\section{REFERENCES}

1 Cystic Fibrosis Foundation. Cystic Fibrosis Foundation Patient Registry, 2011 Annual Data Report. Bethesda, Maryland, 2012.

2 FitzSimmons SC. The changing epidemiology of cystic fibrosis. Journal of Pediatrics 1993;122:1-9.

3 O'Connor GT, Quinton HB, Kahn R, et al. Case-mix adjustment for evaluation of mortality in cystic fibrosis. Pediatr Pulmonol 2002;33:99-105.

4 Quinton H. Using data to identify opportunities for change and to monitor progress. Pediatr Pulmonol 2004;38 (S27):124-1255.

5 Herman DR, Dangler TL, Houser DA, et al. Lewis TG, Masten JL, Taylor IE. The critical phase inspection process: a benchmarking study in search of industries' best practices. Quality Assurance (San Diego, Calif) 1993;2:186-90.

6 Mohr JJ, Mahoney CC, Nelson EC, et al. Batalden PB, Plume SK. Improving health care, Part 3: clinical benchmarking for best patient care. Jt Comm J Qual Improv 1996;22: 599-616.

7 Corey M, McLaughlin FJ, Williams M, et al Levison H. A comparison of survival, growth, and pulmonary function in patients with cystic fibrosis in Boston and Toronto. J Clin Epidemiol 1988;41:583-91.

8 Johnson C, Butler SM, Konstan MW, et al. Morgan W, Wohl ME. Factors influencing outcomes in cystic fibrosis: a center-based analysis. Chest 2003;123:20-7.

9 Padman R, McColley SA, Miller DP, et al. Infant care patterns at epidemiologic study of cystic fibrosis sites that achieve superior childhood lung function. Pediatrics 2007;119:e531-7. 
10 Johnson JK, Mahoney CM, Nelson EC, et al. Batalden PB, Plume SK. Learning from the Best: Clinical Benchmarking for Best Patient Care. In: Nelson EC, Batalden P, Lazar JS, eds. Practice-based learning and improvement: a clinical improvement action guide: Joint Commission Resources; 2007:65-80.

11 Liou TG, Adler FR, Fitzsimmons SC, et al. Cahill BC, Hibbs JR, Marshall BC. Predictive 5-year survivorship model of cystic fibrosis. Am J Epidemiol 2001;153:345-52.

12 Sawicki GS, Rasouliyan L, McMullen AH, et al. Longitudinal assessment of health-related quality of life in an observational cohort of patients with cystic fibrosis. Pediatr Pulmonol 2011;46:36-44.

13 Borowitz D, Baker RD, Stallings V. Consensus report on nutrition for pediatric patients with cystic fibrosis. J Pediatr Gastroenterol Nutr 2002;35:246-59.

14 Stallings VA, Stark LJ, Robinson KA, et al. Feranchak AP, Quinton H. Evidence-based practice recommendations for nutrition-related management of children and adults with cystic fibrosis and pancreatic insufficiency: results of a systematic review. J Am Diet Assoc 2008;108:832-9.

15 Benson HR. An introduction to benchmarking in healthcare. Radiology Management 1994;16:35-9.

16 Huber TP, Godfrey MM, Nelson EC, et al. Mohr JJ, Campbell C, Batalden PB. Microsystems in health care: Part 8. Developing people and improving work life: what front-line staff told us. Jt Comm J Qual Saf. 2003;29:512-22.

17 Britton LJ, Thrasher S, Gutierrez H. Creating a culture of improvement: experience of a pediatric cystic fibrosis center. J Nurs Care Qual 2008;23:115-20; quiz 21-22.
18 Ernst MM, Wooldridge JL, Conway E, et al. Using quality improvement science to implement a multidisciplinary behavioral intervention targeting pediatric inpatient airway clearance. J Pediatr Psychol 2009;1:14-24.

19 Kraynack NC, McBride JT. Improving care at cystic fibrosis centers through quality improvement. Semin Respir Crit Care Med 2009;30:547-58.

20 McPhail GL, Weiland J, Acton JD, et al. Improving evidence-based care in cystic fibrosis through quality improvement. Arch Pediatr Adolesc Med 2010;164: 957-60.

21 Leonard A, Davis E, Rosenstein BJ, et al. Description of a standardized nutrition classification plan and its relation to nutritional outcomes in children with cystic fibrosis. J Pediatr Psychol 2009;35:116-23.

22 Stern M, Wiedemann B, Wenzlaff P. From registry to quality management: the German Cystic Fibrosis Quality Assessment project 1995 2006. Eur Respir J 2008;31:29-35.

23 Stern M, Niemann N, Wiedemann B, et al. Wenzlaff P. Benchmarking improves quality in cystic fibrosis care: a pilot project involving 12 centres. Int J Qual Health Care 2011;23: 349-56.

24 Stern M. The use of a cystic fibrosis patient registry to assess outcomes and improve cystic fibrosis care in Germany. Curr Opin Pulm Med 2011;17:473-7.

25 Wiedemann B, Steinkamp G, Sens B, et al. Stern M. The German cystic fibrosis quality assurance project: clinical features in children and adults. Eur Respir J 2001;17: 1187-94. 